\title{
The viper fangs: clinical anatomy, principles of physical examination and therapy (a review)
}

\author{
Matteo Oliveri, Eva Čermáková, Zdeněk Knotek \\ University of Veterinary and Pharmaceutical Sciences Brno, Faculty of Veterinary Medicine, \\ Avian and Exotic Animal Clinic, Brno, Czech Republic
}

Received January 7, 2016

Accepted August 31, 2016

\begin{abstract}
The assessment of fangs is a fundamental part of clinical examination of viperid snakes. The long curved venom fang is carried by short, highly mobile maxilla. Short anaesthesia is advised for safe physical examination and radiography of the mouth cavity. The fangs are gently forced outside the fang pocket by passing the bar or forceps on the palato-maxillary arch, and rotating them rostrally shifting the mucosal fold. Functional fangs are periodically shed and several generations of replacement teeth lie behind and beneath each fang. In case of fang fracture, therapy should be limited to flushing with a solution of chlorhexidine or povidone iodine, and topical application of pro-coagulant and antibacterial cream. Therapy of chronic fang inflammation is based on removal of necrotized fang and repeated abundant irrigation of the fang pocket. Treatment of chronic stomatitis consists of flushing with chlorhexidine or povidone iodine, physical removal of the plaques, administration of analgesics and antibiotics (marbofloxacin, enrofloxacin or ceftazidime). Extra-oral surgical approach is the best method for odontogenic abscess removal. A vigorous flushing with sterile saline solution, chlorhexidine and povidone iodine and topical application of antibiotics (antibiotic embedded surgical sponge) is advised. Force feeding of the anorectic patient suffering from fang inflammation is a mandatory part of the standard treatment protocol.
\end{abstract}

Venomous snakes, odontogenic abscess, stomatitis

The structure and dentition of the maxilla is of major importance in natural classification of snakes (Edmund 1969) and broad knowledge of the reptile anatomy is mandatory for veterinarians in clinical practice with reptiles. In the viperids (family Viperidae, subfamilies Crotalinae and Viperinae) the fang is the only teeth present on the maxilla and is covered by a large mucosal fold (fang pocket). When the mouth is closed the fang lies folded backwards in a sheath along the roof of the mouth in area of diastema (O'Malley 2005). The long curved venom fang is carried by a relatively short maxilla, which is highly mobile and allows for a broad rotation of the fang. The fang is arranged transversely on the maxilla (Edmund 1969) and is retracted by muscular action during the bite. Fangs in vipers (Viperinae) and pit vipers (Crotalinae) are highly modified fangs (solenoglyphous), have a smooth surface and host the venom canal (VC). This canal communicates with the outside through two orifices, proximal oval foramen and distal oval foramen (Zahradnicek et al. 2008). Venom passes from the venom gland into the fangs entering the proximal oval foramen and is delivered through the distal foramen into the prey (Jackson 2002; Zahradnicek et al. 2008). The triangular maxilla contacts dorsally the prefrontal bone, caudally the ectopterygoid bone and cranially the septomaxilla. The prefrontal bone articulates with the braincase in a movable saddled joint. The snout shows a high degree of mobility, rotating on its attachment on the braincase in concordance with the palatal movements (Deufel and Cundall 2006). The shortness of the palatine bone, together with the mobility of its connection with the maxilla and the snout (composed

Address for correspondence:

Dr. Matteo Oliveri

Avian and Exotic Animal Clinic

Faculty of Veterinary Medicine

University of Veterinary and Pharmaceutical Sciences Brno

Palackého tř.1946/1 61242 Brno, Czech Republic

Phone:
E-mail: matteoloong@hotmail.it
http://actavet.vfu.cz/ 
by the nasals, vomers, septomaxillae, and premaxillae), facilitates the protraction of the palato-maxillary arch during the bite. The eversion of the fang is accomplished through the rotation of the concave surface of the maxilla on the rounded ventral process of the prefrontal (Cundall 1983; Deufel and Cundall 2006) and extrusion of the fang during the bite is accomplished through the action of the dorsal constrictor muscles (DCM) and the protractor pterigoydei muscle (PPM). Modification of the DCM facilitates the striking mechanism, which involves the rotation of the palato-maxillary arch and the eversion of the fang. In viperids the PPM originates on the braincase rostrally compared to other members of the Colubroidea. Located directly caudal to the snout, the PP greatly increases the velocity of the protrusion of the palato-maxillary arch (Cundall 2002). The contraction of PPM and DCM results in the movement of the prefrontal bone that carries the maxilla rostrally and dorsally. The dynamics of the bite involve the extrusion of the fangs and a rapid strike with the head. An angle of approximately $80^{\circ}$ is reached between the braincase and the tip of the fang, as the snake hits the objective (Young et al. 2001; Cundall 2002). Fangs are involved not only in biting the prey but also in the food ingestion. Muscular movements anchor the fangs on the body of the prey, and push it through the pharynx to the entrance of the esophagus (Plate I, Fig. 1). When the snake withdraws its head it extends completely the palato-maxillary arch to free the fangs, reaching an angle of about $90^{\circ}$ to $120^{\circ}$ (Cundall 2002). The maximum extension of the fangs is achieved during the release of the prey. Functional fangs are periodically shed and replaced by a successive generation (Deufel and Cundall 2006). In Trimeresurus albolabris eight generations of venom fangs are present at the same time on the maxilla; seven calcified fangs serve as the replacement for the functional one (Zahradnicek et al. 2008). Before the functional tooth is shed, its neighbor becomes ankylosed and connected with the venom duct. Replacement of the fangs appears to be rapid, and several generations of replacement teeth lie behind and beneath each fang (Edmund 1969).

\section{Principles of physical examination}

The assessment of the health status of the fangs is a fundamental part of the physical examination of the viperid patient. Once the mouth is opened gently with a small rounded bar or forceps (iron or plastic), the mucosa of oral cavity shall be inspected (Plate I, Fig. 2). The fangs are gently forced outside the fang pocket by passing the bar or forceps on the palato-maxillary arch, and rotating them rostrally, shifting the mucosal fold (Fig. 2). The mucosal fold is thin, and there is no accumulation of mucous present in a healthy fold. The health condition and structure of fangs and maxilla can be controlled using radiography on sedated patient. Short anaesthesia for a more detailed physical examination and radiography of the mouth cavity in viperid snakes is recommended.

\section{Therapeutic approach to acute fang inflammation}

The structure of the venom fangs is remarkably fragile, and traumatic injuries are among the most common illnesses involving the fangs in vipers and pit vipers. Clinical signs of acute fang inflammation include anorexia, mouth swelling (Plate II, Fig. 3), ptyalism and haemorrhage. In case of fang(s) fracture, therapy should be limited to flushing with solution of diluted chlorhexidine or povidone iodine and topical administration of procoagulant and antibacterial cream. Local application of hyaluronic acid (spray or drops) can fasten the healing process and reduces pain. As the venom fangs are replaced periodically, the broken fang will ultimately be substituted by a new one. If no complications occur, systemic antibiotics and analgesia are not necessary. 


\section{Therapeutic approach to chronic fang inflammation}

Untreated complicated fracture of the venom fangs are among the most common causes of chronic inflammation of the mucosal fang pocket and prefrontal-maxillary articulation. Possible consequences include necrosis of the root of the fang and periodontitis. Necrotized fangs are brownish and opaque and show a blackish colour at level of the dental root. Diffuse periodontitis should be confirmed or excluded with radiography of the palatine arch. Symptoms of chronic fang inflammation include anorexia, weight loss, apathy, ptyalism and hissing sounds during respiration (Plate II, Fig. 4). The fang pocket shows variable degrees of inflammation and hyperaemia. Profuse and thick mucus may also be present. Therapy is based on removal of necrotized fangs and repeated abundant irrigation of the fang pocket with diluted solution of chlorhexidine or povidone iodine (Plate III, Figs 5, 6). Removal of the necrotized fangs and accurate inspection of the fang pocket are performed on sedated patient. Analgesia is reached with the non-steroidal antiinflammatory drug (NSAID) meloxicam (0.3-0.5 mg/kg, q 24-48 h; Metacam, Boehringer Engelheim, Germany) administered intramuscularly, and topical application of hyaluronic acid or a lidocaine-based spray. Hartmann forceps or haemostats are used to gently remove the fang. Antibacterial prophylaxis involves intramuscular administration of marbofloxacin (10 mg/kg, q 24-48 h, Marbocyl, Mérial, France) for prevention of secondary bacterial infection. Supportive treatment includes fluid therapy $(20-30 \mathrm{ml} / \mathrm{kg}, 0.9 \%$ Natrium chloratum, q $24 \mathrm{~h}$, subcutaneously). Post-operative treatment of chronic fang inflammation should be continued for a minimum of 14 days.

\section{Therapeutic approach to chronic stomatitis}

Chronic stomatitis can result from an untreated fang inflammation (Mehler and Bennett 2006). Diffuse plaques are often seen on the oral cavity. The fang pocket provides a space for proliferation of gram negative bacteria. Clinical symptoms include anorexia, weight loss, open mouth breathing, ptyalism, hissing sounds during respiration, loss of teeth (including venom fangs), and occasional haemorrhages. Stomatitis can progress to involve the nasolacrimal duct (Mehler and Bennett 2006), resulting in sub-spectacular fluid retention, conjunctivitis and panophthalmitis. Stomatitis is a severe disease in snakes, and should be treated aggressively with antibiotics (topically and systemically) and analgesics. Periodontitis should be confirmed or excluded with radiography of the palatine arch. Oral inspection (including examination of fang pockets and removal of necrotized fangs), is performed on an anaesthetised patient. Treatment consists of flushing with chlorhexidine or povidone iodine, physical removal of the plaques, topical administration of analgesics (hyaluronic acid or lidocaine) and systemic administration of NSAIDs and opioids $(10 \mathrm{mg} /$ kg tramadol, Tramal Estada, Germany, q 48-72 h, intramuscularly,) in combination with antibiotics (marbofloxacin, enrofloxacin or ceftazimide) for the first 6 to 7 days. Force feeding of the anorectic patient is a mandatory part of the standard treatment protocol.

\section{Therapeutic approach to odontogenic abscess}

Odontogenic abscess results from untreated chronic inflammation and necrosis of the fang, and is defined as a pus accumulation in a confined cavity, followed by the disintegration of the tissue (Mader 2006). Clinical symptoms of odontogenic abscess include more or less severe deformation of the head and lips, sub-spectacular swelling, anorexia, apathy, weight loss, and neurological symptoms. Standard surgical extra-oral approach is the best method for the surgical removal of the abscess. The incision allows the complete removal of the capsule and accurate sterile swabbing for bacteriology (Plate IV, Fig. 7). Special care 
should be taken dealing with Crotalinae to avoid the pit damage. Vigorous flushing with sterile saline, chlorhexidine and povidone iodine, and topical application of antibiotics (antibiotic embedded surgical sponge) is advised.

\section{Conclusion}

The fangs and the palato-maxillary arch are extremely fragile and specialized structures of viperids. Exploration of the mouth should be routinely performed during clinical examination of Viperinae and Crotalinae. Minor injuries are common, and if left untreated, they can lead to severe problems such as generalized inflammation and stomatitis. The therapeutic approach involves, according to the severity of the case, the use of topic antibiotics and disinfectant, the application of local or systemic analgesics, and surgery.

\section{References}

Cundall D 1983: Activity of head muscles during feeding by snakes: a comparative study. Am J Zool 23: $383-396$

Cundall D 2002: Envenomation strategies, head form, and feeding ecology in vipers. In: Schuett GW, Hölmggren M, Douglas ME, Greene HW (Eds): Biology of the Vipers: Eagle Mountain Publishing, pp 149-162

Edmund AG 1969: Dentition. In: Gans C (Ed.): Biology of the Reptilia. Vol. 1, Morphology A: Academic Press London, pp 117-200

Deufel A, Cundall D 2006: Functional plasticity of the venom delivery system in snakes with a focus on the poststrike prey release behavior. Zool Anz 245: 249-267

Jackson K 2002: How tubular venom-conducting fangs are formed. J Morphol 252: 291-297

Mehler SJ, Bennett AR 2006: Upper alimentary tract disease. In: Mader DR (Ed.): Reptile Medicine and Surgery. Elsevier Saunders, St. Louis, pp 924-930

Mader DR 2006: Abscesses. In: Mader DR (Ed.): Reptile Medicine and Surgery. Elsevier Saunders, St. Louis, pp 715-719

O’Malley B 2005: Snakes. In: O’Malley B (Ed.): Clinical Anatomy and Physiology of Exotic Species. Elsevier Saunders, London, pp 77-93

Young BA, Blair M, Zahn K, Marvin J 2001: Mechanics of venom expulsion in Crotalus, with special reference to the role of the fang sheath. Anat Rec 264: 415-426

Zahradnicek O, Horacek I, Tuckera AS 2008: Viperous fangs: Development and evolution of the venom canal. Mech Develop 125: 786-796 
Plate I

Oliveri M. et al.: The viper fangs: ... pp. 247-250

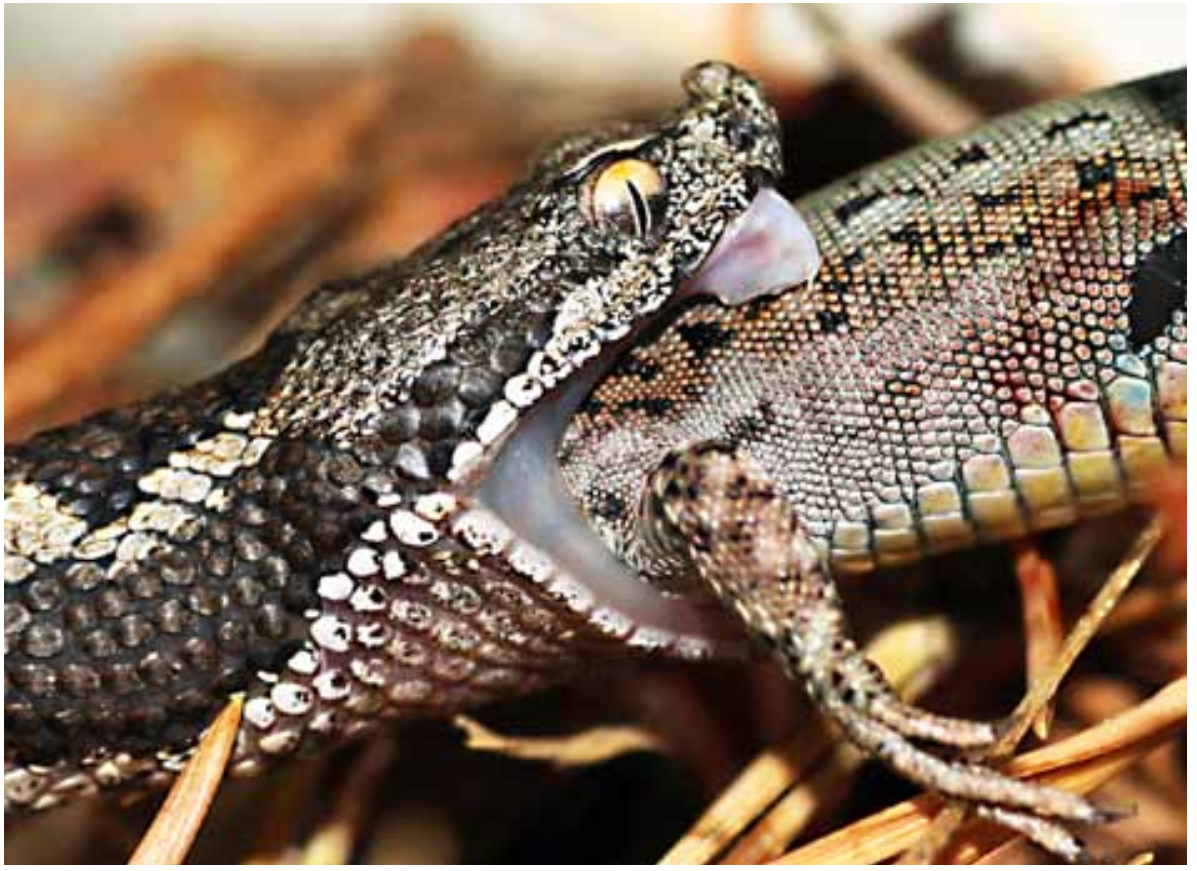

Fig. 1. Vipera latastei latastei ingesting Podarcis hispanca - fangs involved in the food ingestion. Muscular movements anchor the fangs on the body of the prey, and push it through the pharynx to the entrance of the oesophagus. (Photograph courtesy Luis Ruiz Carrasco).
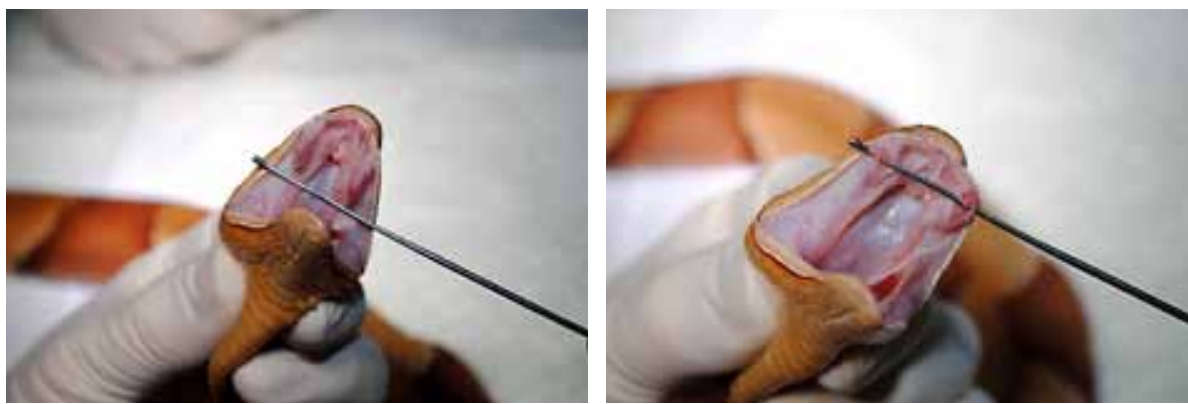

Fig. 2. Agkistrodon contortrix laticinctus. Left: the mouth is opened with a small rounded iron bar and the whole oral mucosa inspected together with the main structure of the mouth. Right: the fang is exposed passing the iron bar on the palato-maxillary arch, moving the mucosal fold that covers them, and rotating the fang rostrally. The normal viperid fang is smooth and diaphanous in appearance. 
Plate II

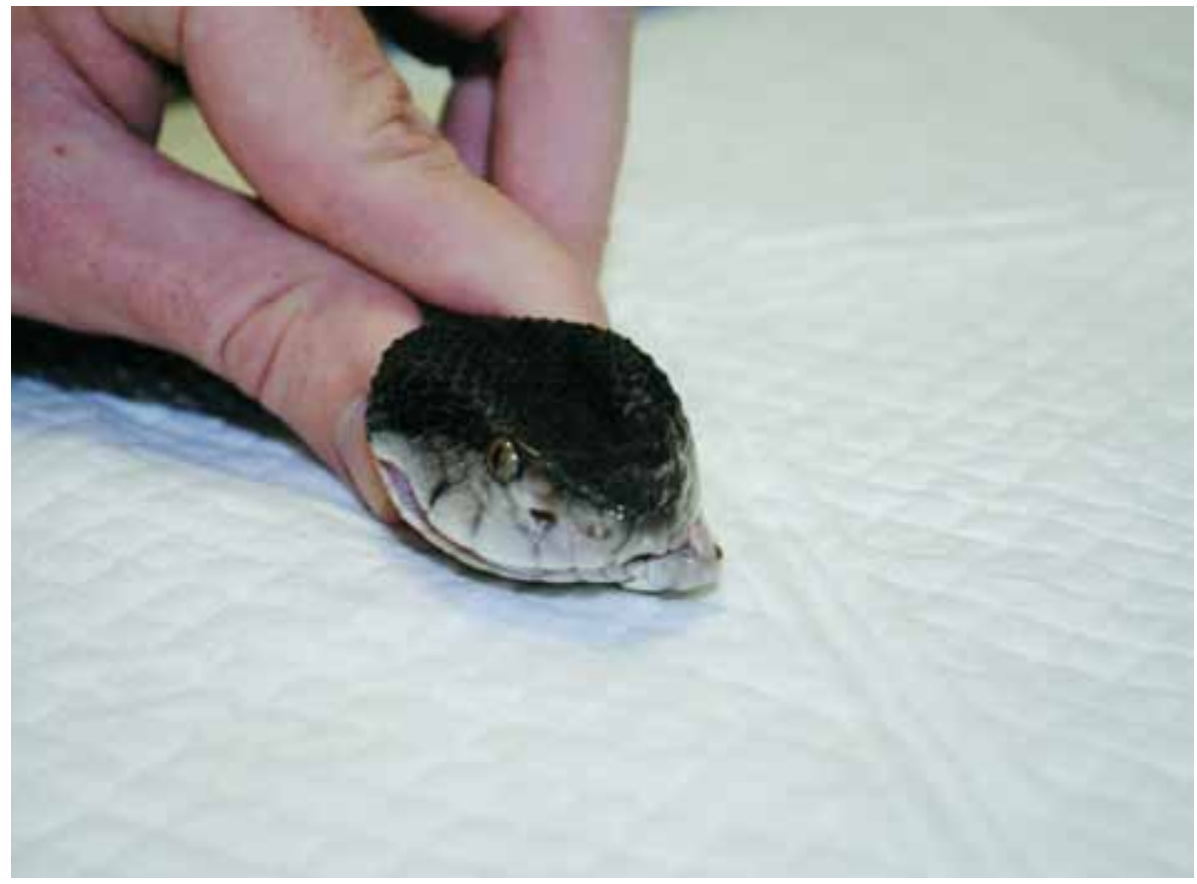

Fig. 3. Bothrops jararaka - swollen mouth secondary to chronic inflammation.

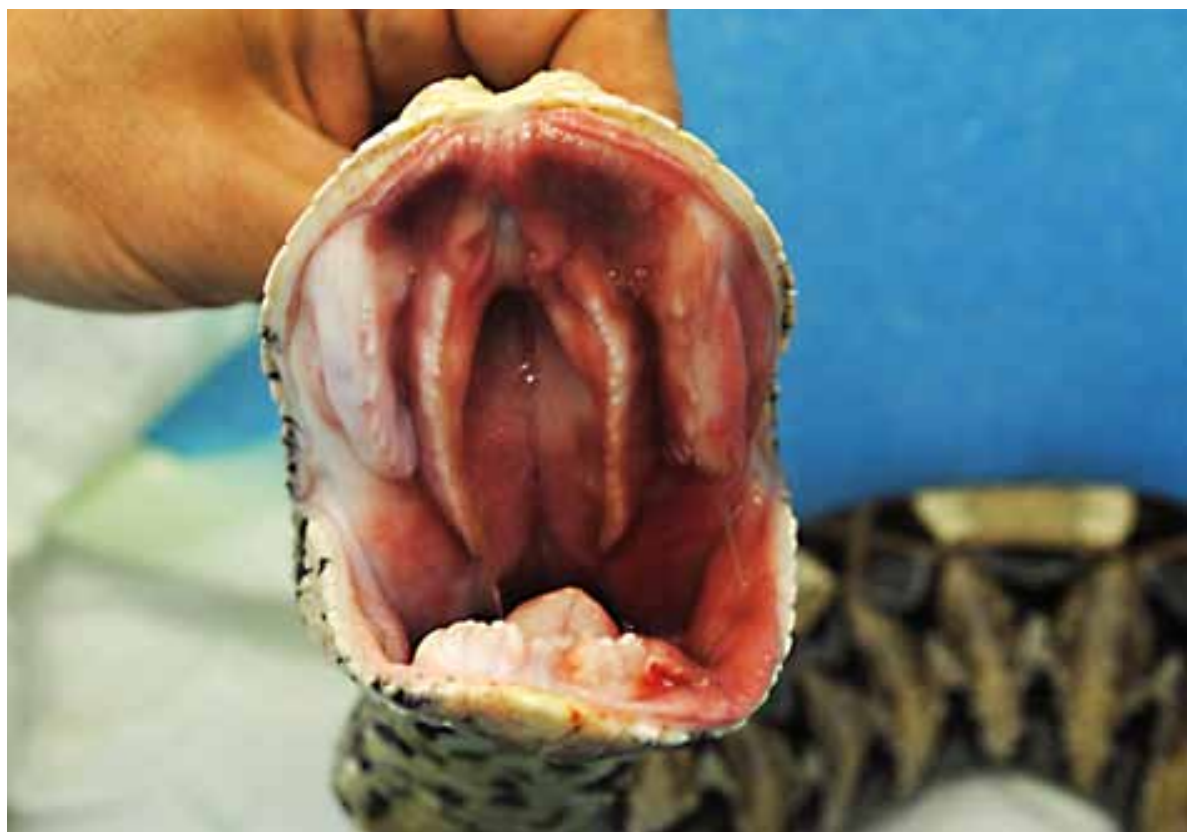

Fig. 4. Bitis gabonica gabonica (animal sedated) - chronic fang inflammation. The right fang pocket is hyperaemic and inflamed. 

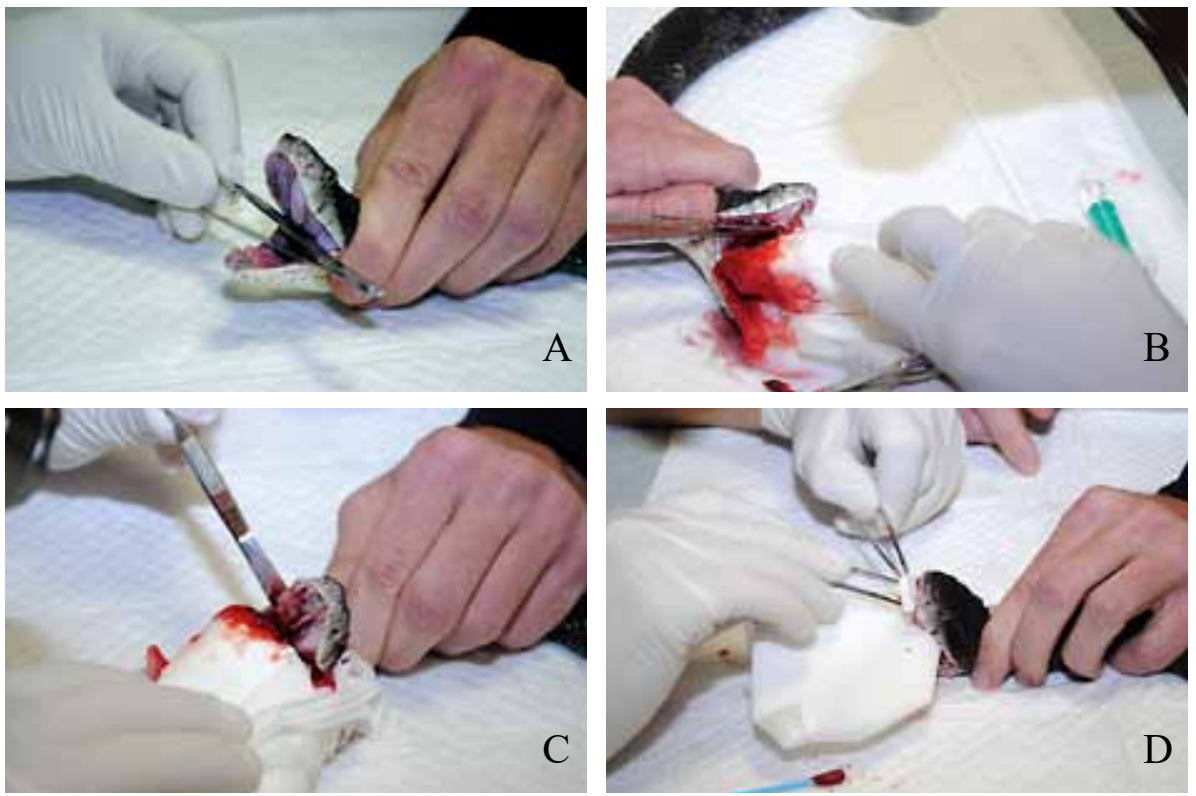

Fig. 5. Bothrops jararaka (same specimen as in Fig. 3). A: clinical examination of the mucosal fold covering the right fang; B: probing the fang pocket causes profuse bleeding; C: several broken fangs are found and removed, and the fold is flushed with saline and povidone iodine; D: haemorrhage is stopped using a surgical collagen sponge.
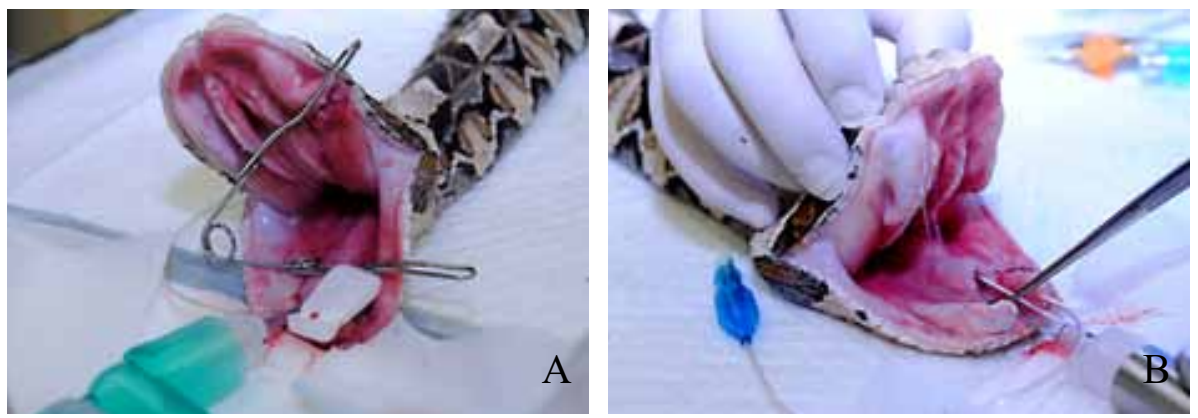

Fig. 6. Bitis gabonica gabonica (same specimen as in Fig. 4). Removal of the necrotized fangs and accurate inspection of the fang pocket performed in general anaesthesia. A surgical sponge (Parasorb, Resorba, Nurnberg, Germany) is used to stop the haemorrhage after biopsy sampling. 
Plate IV
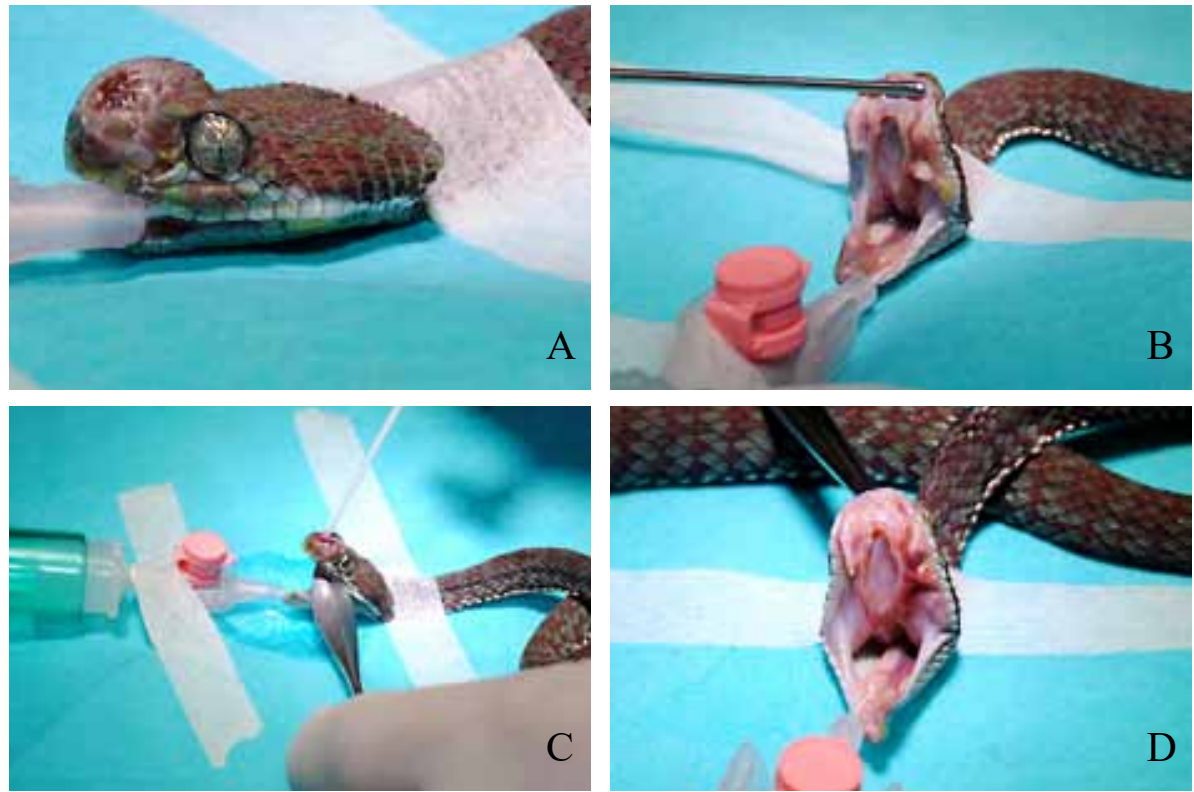

Fig. 7. Trimeresurus venustus - odontogenic abscess. A: big abscess causing deformation of the head and lips; B: plaques of pus, severe inflammation and necrosis of the left fang pocket; C: surgical removal of the abscess through extra-oral approach; D: the left fang pocket after surgical debridement, the venom fang was enclosed into the necrotic tissue. 